\title{
Perfil Microbiológico de Aislamientos en Unidades Neonatales en un Hospital de Tercer Nivel de Bogotá, Colombia
}

\author{
Yolanda Cifuentes ${ }^{1}$, Ariel Ruiz ${ }^{2}$, Aura Leal ${ }^{3}$, Liliana Muñoz ${ }^{4}$, Maria Herrera ${ }^{5}$ \\ y Luz Jiménez 6 \\ ${ }_{1}^{1}$ Médica. Especialista en Pediatría y Neonatología. Departamento de Pediatría, Facultad de \\ Medicina, Universidad Nacional de Colombia. Instituto Materno Infantil. E-mail: \\ yoli52@hotmail.com \\ ${ }^{2}$ Médico. Especialista en Ginecobstetricia, Especialista en Biología de la Reproducción. M. \\ Sc. Departamento de Obstetricia y Ginecología, Centro de Epidemiología Clínica, Facultad de \\ Medicina, Universidad Nacional de Colombia. E-mail: airuizp@unal.edu.co; \\ arielruiz@supercabletv.net.co \\ ${ }^{3}$ Médica. M. Sc. Departamento de Microbiología, Facultad de Medicina, Universidad \\ Nacional de Colombia. E-mail: allealc@unal.edu.co \\ ${ }^{4}$ Bacterióloga. M. Sc. Universidad Colegio Mayor de Cundinamarca. E-mail: \\ lilimunozm@hotmail.com \\ ${ }^{5}$ Bacterióloga. Universidad Colegio Mayor de Cundinamarca. E-mail: mthm@etb.net.co \\ ${ }^{6}$ Bacterióloga. Instituto Materno Infantil.Email: andrejack15@hotmail.com
}

Recibido 30 Enero 2005/Enviado para Modificación 15 Mayo 2005/Aceptado 8 Junio 2005

\section{RESUMEN}

Objetivos Investigar la prevalencia y sensibilidad de gérmenes aislados en recién nacidos hospitalizados en un hospital de referencia de Bogotá. Proponer un esquema antibiótico inicial en infecciones neonatales.

Métodos Se analizaron los cultivos para aerobios y aerobios facultativos practicados entre febrero y diciembre del 2002. Los hemocultivos se procesaron en BacT/ALERT (Dirham, NC); los urocultivos en UROCULT (BioBacter) y las puntas de catéter en Tioglicolato. La identificación se hizo con BBL CRYSTAL (BD, Sparks, MD). La sensibilidad se determinó por difusión de disco.

Resultados Fueron positivos 1097 de 3710 cultivos; se aislaron 64,3\% Gram-positivos, 30,6 \% Gram-negativos y 4,9 \% Candidas. Los Gram-positivos aislados fueron: estafilococos coagulasa negativa (64,2 \%); Enterococcus $(13,8 \%)$ y estafilococos coagulasa positiva $(13,3 \%)$. Los Gram-negativos mas frecuentes fueron Klebsielas (45,2\%); Escherichia coli $(30,9 \%)$ y Serratias $(10,1 \%)$. El $64 \%$ de los estafilococos coagulasa negativos fueron Stafilococcus epidermidis. La sensibilidad del S. epidermidis y los estafilococos coagulasa positivos a la vancomicina fue del $100 \%$. Hubo $86,4 \%$ (IC95\%: 82,3-89,9) de resistencia de los estafilococos coagulasa negativos a los beta-lactámicos. La sensibilidad de los Gram-negativos fue del 98,1 \% 
(IC95\%: 89,9-99,9) a imipenem, 78,1 \% (IC95\%: 64,9-88,2) a gentamicina y 46,6 \% (IC95\%: 28,3-65,7) a amikacina.

Conclusiones Se encontró una alta prevalencia de estafilococos coagulasa negativos particularmente de $S$. epidermidis. No se observó resistencia de S. epidermidis ni estafilococos coagulasa positivos a vancomicina. Se observa resistencia creciente de los estafilcocos coagulasa negativos a oxacilina y de los Gram-negativos a amikacina

Palabras Clave: Stafilococcus epidermidis, infecciones estafilocócicas, recién nacido, infección hospitalaria, tests de sensibilidad microbiana (fuente: DeCS, BIREME).

\section{ABSTRACT \\ Microbiological profiling of isolates from the Neonatal Unit of a third- level hospital in Bogotá, Colombia}

Objectives Investigating the prevalence and sensitivity of germs isolated from newborn in a referral hospital in Bogotá. Suggesting an empirical antibiotic treatment for neonatal infection.

Methods Cultures taken between February and December 2002 were analysed. Blood cultures were processed using BacT/ALERT (Durham, NC), urine cultures by UROCULT (Bio-Bacter) and catheter tips in thioglycollate. BBL CRYSTAL identification system (BD, Sparks, MD) was used for identifying germs. Antibiotic sensitivity was determined by disk diffusion.

Results There were 1,097 positive aerobic and facultative aerobic germ cultures; 64,3\% were Gram-positive, 30,6\% Gram-negative and 4,9\% were yeasts. Gram-positive germs consisted of coagulase-negative staphylococci $(64,2 \%)$, enterococcus $(13,8 \%)$ and coagulase-positive staphylococci $(13,3 \%)$. The most frequent Gram-negatives were Klebsiella $(45,2 \%)$, Eschericha coli $(30,9 \%)$ and Serratia $(10,1 \%)$. Staphylococcus epidermidis accounted for $64 \%$ of the coagulase-negative staphylococci. S. epidermidis susceptibility to vancomycin was $100 \%$. Coagulase-negative staphylococci susceptibility to rifampin and amikacin was $59 \%$ and $67,4 \%$ (respectively). Coagulase-negative staphylococci resistance to beta-lactams was $86,4 \%$ (95\% Cl: 82,3-89,9). Coagulase-positive staphylococci sensitivity to vancomycin was 100\%. Gram-negative susceptibility to imipenem was $98,1 \%$ (95\% Cl: $89,9-99,9), 78,1 \%$ to gentamicin $(95 \% \mathrm{Cl}: 64,9-88,2)$ and $46,6 \%$ to amikacin (95\% Cl: 28,3-65,7).

Conclusions There was high coagulase-negative staphylococci prevalence in neonatal infection (particularly S. epidermidis). All S. epidermidis and coagulase-positive staphylococci were sensitive to vancomycin. There was increasing coagulase-negative staphylococci and Gram-negative resistance to oxacillin and amikacin, respectively.

Key Words: Staphylococcus epidermidis, infection, staphylococci, infant, newborn, cross-infection, microbial sensitivity test (source:MeSH, NLM). 
$\mathrm{L}$ as infecciones causadas por bacterias y hongos constituyen una causa muy importante de morbimortalidad a nivel mundial, afectando anualmente a más de 200.000 individuos en los Estados Unidos. Son mas frecuentes en neonatos pretérmino y en países en vías de desarrollo. Los estudios de la National Nosocomial Infection Surveillance y del Surveillance and Control of Pathogens of Epidemiologic Importance muestran que los estafilococos coagulasa negativa (ECN) son los patógenos más prevalentes en las infecciones nosocomiales $(1,2)$. También se ha informado un incremento de las infecciones por cándida y pseudomona, un aumento en la resistencia de los estafilococos a la oxacilina, de los estreptococos a la penicilina y de los enterococos a la vancomicina (3-5).

Un estudio sobre 662 episodios de sepsis neonatal nosocomial, mostró que los Gram-positivos se aislaron en el 58,4 \% de los casos, siendo el Staphylococcus epidermidis el más frecuente ( $42 \%$ ); los Gram-negativos mas frecuentes fueron Escherichia coli y Klebsiella y se aislaron hongos, fundamentalmente Candida sp, en el $12 \%$ de los episodios (6).

El presente estudio es un análisis retrospectivo de los cultivos para aerobios y aerobios facultativos, obtenidos en pacientes hospitalizados en unidades neonatales de un hospital maternoperinatal de referencia. Los objetivos de estudio fueron: 1) identificar los microorganismos más frecuentemente aislados en neonatos hospitalizados en el Instituto Materno Infantil (IMI) de Bogotá, Colombia; 2) investigar el perfil de resistencia a los antibióticos de los gérmenes aislados y 3 ) proponer un esquema antibiótico inicial para el manejo de infecciones neonatales.

\section{MATERIALES Y MÉTODOS}

Se analizaron retrospectivamente todos los resultados consecutivos de los cultivos practicados entre el 1 de febrero y el 31 de diciembre del 2002, de muestras obtenidas de recién nacidos hospitalizados en el IMI, institución universitaria de referencia y único hospital exclusivamente perinatal en Colombia. Todos los resultados positivos fueron elegibles para el estudio. Durante el periodo hubo 2331 ingresos a la Unidad de Neonatología; 1057 neonatos remitidos y 1274 niños nacidos en el IMI.

Las muestras para hemocultivo se incubaron en el sistema automatizado (BacT/ALERT; Biomeriux, Durham, NC) urocultivos en UROCULT (BioBacter) y las puntas de catéter, las muestras provenientes de secreciones y 
líquido cefalorraquídeo (LCR) en medio enriquecido de caldo Tioglicolato para posteriormente subcultivarlas. Las muestras de LCR se sembraron además en agar sangre y agar chocolate. Los subcultivos se procesaron en agar tripticasa soya al $5 \%$ en sangre de cordero y en medio de McConkey. Para la identificación de los microorganismos Gram-positivos y Gram-negativos se utilizó BBL CRYSTAL (Becton Dickinson, Sparks, MD, USA).

La sensibilidad bacteriana se estudió por el método de difusión de disco, para los siguientes antibióticos: penicilina $(30 \mu \mathrm{g})$, oxacilina $(1 \mu \mathrm{g})$, vancomicina $(30 \mu \mathrm{g})$, rifampicina $(5 \mu \mathrm{g})$, gentamicina $(10 \mu \mathrm{g})$, amikacina $(30 \mu \mathrm{g})$, imipenem $(10 \mu \mathrm{g})$, netilmicina $(30 \mu \mathrm{g})$, trimetoprim/sulfa $(23,75 / 1,25 \mu \mathrm{g})$, ampicilina $(20 \mu \mathrm{g})$, ampicilina/sulbactam $(20 \mu \mathrm{g})$ y ciprofloxacina $(5 \mu \mathrm{g})$.

Se hizo análisis descriptivo de los datos e intervalos de confianza utilizando paquete estadístico STATA 6.0. El estudio se ajustó a las normas de investigación con riesgo mínimo en humanos.

\section{RESULTADOS}

En total se realizaron 3710 cultivos para aerobios y aerobios facultativos distribuidos así: 2255 hemocultivos; 751 urocultivos; 271 cultivos de punta de catéter; 202 LCR y 231 cultivos de otras muestras (103 coprocultivos; 55 secreciones oculares; 40 secreciones traqueales; 14 líquidos peritoneales; 9 de tejidos blandos; 6 líquidos pleurales; 2 muestras osteoarticulares; una de jugo gástrico y una de secreción de ileostomía).

Tabla 1. Aislamientos de acuerdo con el tipo de muestra.

\begin{tabular}{lrrrcrrr}
\multicolumn{7}{c}{ Unidad de Neonatología. IMI. } \\
\hline $\begin{array}{c}\text { Tipo de } \\
\text { muestra } \\
(\mathrm{n}=1 \text { 097) }\end{array}$ & \multicolumn{2}{c}{ Gram positivos } & \multicolumn{2}{c}{ Gram negativos } & \multicolumn{2}{c}{ Candidas } \\
\hline Hemocultivos & 486 & 86,8 & 59 & 10,5 & 15 & 2,7 \\
Urocultivos & 20 & 9,3 & 159 & 74,3 & 35 & 16,3 \\
Catéteres & 111 & 87,4 & 16 & 12,6 & 0 & 0 \\
LCR & 5 & 62,5 & 3 & 37,5 & 0 & 0 \\
Otros & 85 & 44,7 & 99 & 53,1 & 4 & 2,1 \\
Totales & 707 & 64,3 & 336 & 30,6 & 54 & 4,9 \\
\hline
\end{tabular}

En 1097 cultivos positivos se identificaron 707 Gram-positivos $(64,3 \%)$; 336 Gram-negativos $(30,6 \%)$ y 54 cándidas $(4,9 \%)$. La Tabla 1 señala su distribución por tipo de muestra. Los Gram-positivos aislados fueron: ECN 
(64,2\%); Enterococcus (13,9\%); Estafilococos Coagulasa Positivos (ECP) (13,3\%); Streptococcus (3,7\%); Micrococcus (2,5\%); Lactococcus $(1,7$ $\%)$; bacilos Gram-positivos $(0,4 \%)$ y Corynebacterium $(0,3 \%)$. Los Gramnegativos fueron: Klebsielas $(45,2 \%)$; E. coli $(30,9 \%)$; Serratias $(10,1 \%)$; Enterobacter (5,9\%); Pseudomonas (2,1\%); Acinetobacter (1,8 \%); Proteus $(1,5 \%)$ y otros $(2,4 \%)$.

Hemocultivos

Se identificaron 486 (86,8 \%) Gram-positivos; 59 (10,5\%) Gram-negativos y $15(2,7 \%)$ cándidas. No se diferenció si la infección correspondía a infección neonatal temprana o tardía. Los Gram-positivos aislados fueron: ECN (72\%); ECP (13,4 \%); Enterococcus (6,6\%); Micrococcus (3,1\%); Streptococcus $(2,5 \%)$; Lactococcus $(1,6 \%)$; bacilos Gram-positivos $(0,6 \%)$ y Corynebacterium $(0,2 \%)$. Los ECN correspondieron en un $65,1 \%$ a $S$. epidermidis. En la Tabla 2 se presenta la sensibilidad de ECN, S. epidermidis y ECP. Los Gram-negativos fueron: Klebsiellas $(45,8 \%)$, E. coli $(23,7 \%)$, Serratia $(11,9 \%)$ y otros $(18,6 \%)$. La Tabla 3 muestra la sensibilidad de los Gram negativos, Klebsiella y E. coli.

Tabla 2. Sensibilidad en porcentaje (IC95\%) $)^{\text {a }}$ de los SCN, del S. epidermidis y de los SCP aislados de hemocultivos Unidad de Neonatología. IMI.

\begin{tabular}{lccc}
\hline \multicolumn{1}{c}{ Antibiótico } & SCN & S. epidermidis & SCP \\
\hline Penicilina & $0,9(0,2-2,6)$ & $0,5(0,0-2,6)$ & $7,5(2,1-18,2)$ \\
Oxacilina & $13,6(10,1-17,7)$ & $14,8(10,4-20,1)$ & $36,9(25,3-49,8)$ \\
Vancomicina & $99,4(97,9-99,9)$ & $100(98,4-100)^{\mathrm{b}}$ & $100(94,5-100)^{\mathrm{b}}$ \\
Rifampicina & $59,2(52,9-65,2)$ & $53,3(45,4-60,9)$ & $88,2(76,1-95,6)$ \\
Gentamicina & $37,1(30,6-43,9)$ & $34(26,3-42,2)$ & $66,7(46-83,5)$ \\
Amikacina & $67,4(61,9-72,5)$ & $64,9(58,2-71,3)$ & $58,6(44,9-71,4)$ \\
\hline a'Entre paréntesis: Intervalo de confianza del $95 \%{ }^{\text {b }}$ Intervalo de confianza del $97,5 \%$, \\
unilateral
\end{tabular}

Urocultivos

Se aislaron 159 Gram-negativos (74,3\%); 20 Gram-positivos (9,3\%) y 35 cándidas (16,3\%). Los Gram-negativos aislados fueron: Klebsiella (46,5\%), E. coli $(37,7 \%)$, Serratia $(8,2 \%)$, Enterobacter $(4,4 \%)$, Proteus $(1,2 \%)$ y otros $(1,9 \%)$. La sensibilidad de klebsiella a imipenem, gentamicina y amikacina fue del 98,5 \% (IC95\%: 91,9-99,9), 69,5 \% (IC95\%: 57,3-80,1) y 19,1 \% (IC95\%: 9,1-33,3) respectivamente. La sensibilidad de E. coli a imipenem, gentamicina y amikacina fue del $100 \%$ (IC95\%: 93,3-100), 77,1\% (IC95\%: 64,2-87,3) y 73,5\% (IC95\%: 55,6-87,1), respectivamente. 
Cultivos de punta de catéter

Se aislaron 111 Gram-positivos $(87,4 \%)$ y 16 Gram-negativos $(12,6 \%)$. Los Gram-positivos fueron: ECN (64\%), de los que el 64,7\% fue S. epidermidis; ECP (16,2 \%) de los que el 83,3 \% fue S. aureus; Enterococcus $(15,3 \%)$ y otros $(4,5 \%)$. Los Gram-negativos aislados fueron: Klebsiella (37,5\%); Serratia (31,2\%); E. coli (12,5\%); Enterobacter (12,5\%) y Pseudomonas $(6,2 \%)$.

Tabla 3. Sensibilidad en porcentaje (IC95\%) $)^{\mathrm{a}}$ de los gram negativos, Klebsiella y E. coli aisladas de hemocultivos. Unidad de Neonatología. IMI.

\begin{tabular}{|c|c|c|c|}
\hline Antibiótico & Gram-negativos & Klebsiella & E. Coli \\
\hline Imipenem & $98,1(89,9-99,9)$ & $96,1(80,4-99,9)$ & $100(73,5-100)^{b}$ \\
\hline Gentamicina & $78,2(64,9-88,2)$ & $88(68,8-97,5)$ & $85,7(57,2-98,2)$ \\
\hline Amikacina & $46,7(28,3-65,7)$ & $30(6,7-65,2)$ & $70(34,8-93,3)$ \\
\hline Netilmicina & $46,1(26,6-66,6)$ & $18,7(4-45,6)$ & $100(29,2-100)^{b}$ \\
\hline Ampicilina/Sulbactam & $38,4(25,9-53,1)$ & $29,2(12,6-51,1)$ & $69,2(38,6-90,9)$ \\
\hline Ceftriaxona & $44,7(28,6-61,7)$ & $25(8,7-49,1)$ & $60(26,2-87,8)$ \\
\hline Ciprofloxacina & $94,9(82,7-99,4)$ & $95,2(76,2-99,9)$ & $100(66,4-100)^{b}$ \\
\hline
\end{tabular}
$\mathrm{ral}$

\section{DISCUSIÓN}

Se presentan los resultados de 3710 cultivos para aerobios y aerobios facultativos, de muestras obtenidas de neonatos hospitalizados. En total 1097 cultivos fueron positivos $(29,5 \%)$. En hemocultivos y cultivos de punta de cateter predominaron los aislamientos de Gram-positivos $(86,7 \%$ y $87,4 \%$, respectivamente), mientras que en los urocultivos se aislaron mas frecuentemente Gram-negativos $(74,3 \%)$. Estos hallazgos concuerdan con la literatura $(6,7)$.

En el 86,8 \% de los hemocultivos positivos se aislaron Gram-positivos, el $72 \%$ fueron ECN y de éstos, el $65 \%$ eran S. epidermidis, indicando que este germen constituye un problema importante en las unidades de neonatología del IMI. Pessoa-Silva y colaboradores encontraron que la mortalidad específica atribuible a S. epidermidis era del $24 \%$ en neonatos con sepsis tardía (10). Otros autores también han encontrado un predominio de los ECN en neonatos con sepsis tardía $(8,9)$. Sin embargo, en otros estudios la mayoría de los aislamientos de hemocultivos en neonatos con septicemia correspondió a bacilos Gram-negativos $(11,13,14)$. 
En este estudio el 99,4 \% (IC95\%: 97,9-99,9) de los ECN y el $100 \%$ (IC97,5\%: 98,4-100) de los S. epidermidis de hemocultivos fueron sensibles a vancomicina. La sensibilidad de los ECN a rifampicina y amikacina fue solo del 59,1\% (IC95\%: 52,9-65,2) y 67,4\% (IC95\%: 61,9-72,5), respectivamente y la del S. epidermidis fue de 53,2\% (IC95\%: 45,4-60,9) y 64,9\% (IC95\%: 58,2-71,3). La resistencia de los ECN a los $\beta$ lactámicos fue del 86,4 \% (IC95\%: 82,3-89,9). Estos patrones de sensibilidad son similares a los informados por Diekema y colaboradores (5) en aislamientos de infecciones nosocomiales y en la comunidad.

Los S. epidermidis, los ECP y el 99,4 \% (IC95\%: 97,9-99,9) de los ECN fueron sensibles a la vancomicina. Hubo dos aislamientos de ECN resistentes a la vancomicina. El primero, S. Saprophyticus, en un paciente en quien 5 días antes se había aislado un S. Epidermidis sensible a vancomicina y el segundo en un hemocultivo positivo para Serratia marcenses y S. Xylosus por lo puede tratarse de una contaminación o de interferencia en el antibiograma por Serratia.

La sensibilidad de los Gram-negativos asilados en hemocultivos fue del 98,1 \% (IC95\%: 89,9-99,9) al imipenem, del 78,2 \% (IC95 \%: 64,9-88,2) a la gentamicina y del $46 \%$ (IC95\%: 28,3-65,7) a la amikacina. La sensibilidad de la Klebsiella a la gentamicina fue del $88 \%$ (IC95\%: 68,8-97,5) y la de E. coli del 85,7 \% (IC95\%: 57,2-98,2) para ese antibiótico. Aunque fue alta la sensibilidad a ciprofloxacina tanto de klebsiella $(94,9 \%$; IC95\%: 82,7-99,4) como de E. coli (100\%; IC97,5\%: 66,4-100), el uso de este antibiótico está restringido en neonatología a casos de infecciones graves por gérmenes sensibles únicamente a ciprofloxacina.

El 74,3 \% de los gérmenes aislados de urocultivos fueron Gram-negativos, pero llama la atención la frecuencia alta de aislamientos de cándida (16,3 \%). El 98,5 \% (IC95\%: 94,8-99,8) de los Gram-negativos aislados de urocultivos fueron sensibles a imipenem; una cepa de Klebsiella y una de Proteus fueron resistentes a este antibiótico. Solo el 41,7 \% (IC95\%: 31,5$52,6)$ de los Gram-negativos fueron sensibles a la amikacina. La sensibilidad al imipenem fue del 98,5\% (IC95\%: 94,8-99,8) para Klebsiella y $100 \%$ (IC97,5\%: 93,3-100) para E. coli.

Con estos hallazgos, en casos de sepsis neonatal sugerimos implementar un esquema inicial asociando vancomicina, para cubrir S. epidermidis y $\mathrm{SCP}$, con gentamicina, para cubrir los Gram-negativos. Huang y colaboradores también han sugerido incluir la vancomicina en los esquemas empíricos para bacteremia por ECN (15), sin embargo, Rubin y colaboradores sugirie- 
ron desarrollar guías de práctica que disminuyan el uso empírico de éste antibiótico para evitar resistencia (16).

Los gérmenes mas frecuentemente aislados de puntas de catéter fueron los Gram-positivos $(87,4 \%)$, de éstos el $63,9 \%$ correspondieron a ECN, siendo el S. epidermidis el más frecuente. La susceptibilidad de los ECN cultivados a partir de muestras de punta de catéter fue similar a la de los hemocultivos: $100 \%$ (IC97,5\%: 94,9-100) a la vancomicina y 15,6 \% (IC 95\%: 7,8-26,9) a la oxacilina. Desde 1972 se ha informado acerca de la infección por ECN en pacientes con catéteres atribuida a la capacidad de colonización y formación del "biofilm" con persistencia de la infección (17). La disminución de la susceptibilidad a los antibióticos en el biofilm se debería a una combinación de factores como pobre penetración del antibiótico, disminución de nutrientes y crecimiento lento, respuestas adaptativas al estrés, formación de multicapas de defensa, difusión de nutrientes y compromiso de las defensas del hospedero por la presencia de biomateriales (18-20).

No se aisló ninguna cepa de estreptococo beta-hemolítico del grupo B y, al contrario de lo que ocurre en Australia, el Reino Unido y Estados Unidos $(9,21)$, la infección neonatal temprana por este germen no constituye un problema en las unidades neonatales del IMI. No está claro si existen diferencias específicas de prevalencia o si contribuye el uso anteparto de antibióti$\cos (22)$ que constituye una rutina en nuestras pacientes con ruptura prematura de membranas pretérmino.

Una limitación de nuestro estudio es la ausencia de cultivos para anaerobios, ya que no se practican rutinariamente en el IMI.

En conclusión, la mayoría de los aislamientos en las unidades neonatales del IMI fueron Gram-positivos. Se encontró una prevalencia alta de ECN en general $\mathrm{y}$, particularmente, de S. epidermidis. No tuvimos ningún aislamiento de estreptococo del grupo B. Los perfiles de sensibilidad indican que es urgente modificar el esquema antibiótico que se inicia antes de los resultados de los cultivos y que dicho esquema debe incluir a la vancomicina. En los pacientes que reciben vancomicina y tienen infección persistente por $\mathrm{S}$. epidermidis, se deben considerar factores como la presencia de catéteres, pues in vitro no se ha demostrado la resistencia a la vancomicina. Debe prestarse atención a la creciente resistencia a oxacilina de los ECN y a la amikacina de los Gram-negativos •

Agradecimientos. A Sandra L. Díaz, Angélica Amador, Johanna Huertas y Andrea Morales, estudiantes de la Universidad Colegio Mayor de Cundinamarca 


\section{REFERENCIAS}

1. Edmond MB, Wallace SE, McClish DK, Pfaller MA, Jones RN, Wenzel RP. Nosocomial bloodstream infections in United States hospitals: a three-year analysis. Clin Infect Dis 1999; 29: 239-244.

2. Schaberg DR, Culver DH, Gaynes RP. Major trends in the microetiology of nosocomial infection. Am J Med 1991; 91: 72S-75S.

3. Pfaller MA, Jones RN, Doren GV, Kugler R. and The SENTRY Participants Group. Bacterial pathogens isolated from patients with bloodstream infection: Frequencies of occurrence and antimicrobial susceptibility patterns from The Sentry Antimicrobial Surveillance Program (United States and Canada, 1997). Antimicrob Agents Chemother 1998; 42(7): 1762-1770.

4. Raimundo O, Heussler H, Bruhn JB, Suntrarachun S, Kelly N, Deighton MA, et al. Molecular epidemiology of coagulase-negative staphylococcal bacteraemia in a newborn intensive care unit. J Hosp Infect. 2002; 51(1):33-42.

5. Diekema DJ, Pfaller MA, Schmitz FJ, Smayevsky J, Bell J, Jones RN, et al.: Survey of infections due to Staphylococcus species: Frecuency of occurrence and antimicrobial susceptibility of isolates collected in the United States, Canada, Latin America, Europe and the Western Pacific Region for the SENTRY Antimicrobial Surveillance Program, 1997-1999.Clinical Infectious Diseases 2001; 32 (supl 2):S114-32.

6. Lopez JB, Coto D, Fernandez B. Neonatal sepsis of nosocomial origin: an epidemiological study from the "Grupo de Hospitales Castrillo. J Perinat Med. 2002;30(2):149-57.

7. Cunha Ma. de L, Lópes CAM, Rugolo LMSS, Chalita LVS. Clinical significance of coagulase-negative staphylococci isolated from neonates. J Pediatr (Rio J) 2002; 78(4): 279-288.

8. Stoll BJ, Hansen N, Fanaroff AA, Wright LL, Carlo WA, Ehrenkranz RA, et al. Late-onset sepsis in very low birth weight neonates: the experience of the NICHD Neonatal Research Network. Pediatrics. 2002; 110 (2 Pt 1):285-91.

9. Mehr SS, Sadowsky JL, Doyle LW, Carr J. Sepsis in neonatal intensive care in the late 1990s. J Paediatr Child Health. 2002; 38(3):246-51.

10. Pessoa-Silva CL, Miyasaki CH, de Almeida MF, Kopelman BI, Raggio RL, Wey SB. Neonatal late-onset bloodstream infection: attributable mortality, excess of length of stay and risk factors. Eur J Epidemiol. 2001; 17(8):71520.

11. Ahmed AS, Chowdhury MA, Hoque M, Darmstadt GL. Clinical and bacteriological profile of neonatal septicemia in a tertiary level pediatric hospital in Bangladesh. Indian Pediatr. 2002; 39(11):1034-9.

12. Galanakis E, Krallis N, Levidiotou S, Hotoura E, Andronikou S. Neonatal bacteraemia: a population-based study. Scand J Infect Dis. 2002; 34(8):598-601.

13. Nambiar S, Singh N. Change in epidemiology of health care-associated infections in a neonatal intensive care unit. Pediatr Infect Dis J. 2002; 21(9):83942 . 
14. Stoll BJ, Hansen N, Fanaroff AA, Wright LL, Carlo WA, Ehrenkranz RA, et al. Changes in pathogens causing early-onset sepsis in very-low-birth-weight infants. N Engl J Med. 20021 25; 347(4):240-7.

15. Huang SY, Tang RB, Chen SJ, Chung RL. Coagulase-negative staphylococcal bacteremia in critically ill children: risk factors and antimicrobial susceptibility. J Microbiol Immunol Infect. 2003; 36(1):51-5.

16. Rubin LG, Sanchez PJ, Siegel J, Levine G, Saiman L, Jarvis WR; Pediatric Prevention Network. Evaluation and treatment of neonates with suspected lateonset sepsis: a survey of neonatologists' practices. J Perinatol. 2002; 22(7):547-9.

17. O'Gara Jp, Humphreys H. Staphylococcus epidermidis biofilms: importante and implications. J Med Microbiol 2001; 582-587.

18. Stewart PS. Mechanisms of antibiotic resistance in bacterial biofilms. Int J Med Microbiol 2002; 292: 107-113.

19. Lyte M, Freestone PPE, Neal C, Olson B, Haigh R, Bayston R, et al. Stimulation of staphylococcus epidermidis growth and biofilm formation by catecholamine inotropes. Lancet 2003; 361:130-135.

20. Boelens JJ, Dankert J, Murk JL, Weening JJ, van der Poll T, Dingemans KP, et al. Biomaterial-assocated persistence of Staphylococcus epidermidis in pericatheter macrophages. J Infect Dis 2000; 181: 337-49.

21. Luck S, Torny M, d'Agapeyeff K, Pitt A, Heath P, Breathnach A, et al. Estimated early-onset group B streptococcal neonatal disease. Lancet 2003; 361(9373): 1953-4.

22. Towers CV, Briggs GG. Antepartum use of antibiotics and early-onset neonatal sepsis: the next 4 years. Am J Obstet Gynecol. 2002; 187(2):495-500. 